\title{
Extraction Process Optimization of Total Alkaloid from Actinidia arguta
}

\author{
Yang-yang Liu ${ }^{1, a}$, Chang-jiang Liu ${ }^{\star b}$ \\ ${ }^{1}$ College of Food, Shenyang Agricultural University, Shenyang, 110866, China \\ * College of Food, Shenyang Agricultural University, Shenyang, 110866, China \\ ae-mail: juanjuanmao11@163.com be-mail: liucj597@sohu.com
}

Keywords: Actinidia arguta; Total Alkaloids; Ultrasonic extraction; Orthogonal experimental design

\begin{abstract}
To study on optimal conditions for extracting the higher content of total alkaloid from Actinidia arguta fruits, the effects of ethanol concentration, temperature, time and solid-liquid ratio on extraction efficiency total alkaloid were explored. The results showed that the optimum extraction conditions included $70 \%$ ethanol as extracting solution, temperature on 60C, extraction time by $20 \mathrm{~min}$, ratio of solid- liquid on 1:30 $(\mathrm{g} / \mathrm{mL})$. By this method, the total alkaloids of Actinidia arguta fruits were extracted to $1.021 \mathrm{mg} / \mathrm{g}$. This experiment of extraction method is simple and easy to operate. It was improved the extraction yield of the total alkaloid extraction from Actinidia arguta fruits.
\end{abstract}

\section{Introduction}

The Actinidia arguta is an important wild fruit resource. This wild plant distributes in the Northeast China, North China, Shandong, Northwest China and Yangtze valley, and chiefly concentrated in the southern part of the mountainous area in the Northeast [1]. It is rich of many nutrients, such as high content of $\mathrm{V}_{\mathrm{C}}(200-400 \mathrm{mg} / 100 \mathrm{~g})$ [2]. It is an ideal green food and healthy food, the root, stem and fruit are all useful for us because of their medical value and healthy function. The fruits contain lots of SOD, amino acid, sugars and etc. Meanwhile, and have the effect on the hyperlipidemia, hypertension, and stenocardia [3, 4].

In general, alkaloids refer to the natural alkaline nitrogen compounds in plants [5]. Alkaloid has strong physiological role of valuable medicines for people and animals [6]. Alkaloid is the study of a hot spot [7, 8], at present, the researchers have no further research and study in the Actinidia arguta. We can only search the reports of fruit SOD, Amino acids, and Enzymes. This article offers the important value on the total exploitation of Actinidia arguta.

In this paper a new optimal design of extracting of total alkaloids in Actinidia arguta has been studied. The purpose is to improve the peach alkaloid extraction yield. This new extraction process provides a theoretical basis for the resources utilization of the new medicinal plant.

\section{Materials and Methods}

Primary materials and instruments. Fruits of the Actinidia arguta were purchased from the College of Food, Shenyang Agricultural University, China. UV-type 240 spectrophotometer (SHIMADZU Japan). Aconitine (American Sigma), Other reagents are domestic analytical reagents.

Standard curve. Under the condition of certain $\mathrm{pH}$, alkaloids with hydrogen ion combined into salt. Acid dyes and the salt combined into non-ferrous complex. Aconitine has the nature of the total alkaloid. However, total alkaloids of Actinidia arguta determinate by spectrophotography and aconitine as standard. the determination of absorbance values were used the solution from the chloroform layer and joined the $\mathrm{Na}_{2} \mathrm{SO}_{4}$ for dehydration. The regression equation $\mathrm{Y}=0.0682 \mathrm{x}+0.0077, \mathrm{r}=0.9997(\mathrm{n}=6)$.

The sample determination of alkaloids. Using ultrasound, $5 \mathrm{~g}$ of dried powder of samples was extracted with $100 \mathrm{~mL}$ of $80 \%$ ethanol 3 times at $40 \mathrm{C}$ for $30 \mathrm{~min}$. The ethanol extracts were evaporated to dryness by rotary evaporation at $60 \mathrm{C}$ under reduced pressure to produce a ethanol 
crude extract. The crude extract was dissolved by $20 \mathrm{~mL} 2 \% \mathrm{HCL}$ and filter. The same volume of chloroform extraction 2 times, $\mathrm{pH}$ to 10 by ammonia, and use the same volume of chloroform extraction 3 times again. The extracts were evaporated to dryness by rotary evaporation and constant volume to $25 \mathrm{~mL}$. $1 \mathrm{~mL}$ extracts liquid, $6 \mathrm{~mL}$ chloroform and $6 \mathrm{~mL} \mathrm{NaOH}-\mathrm{KHC}_{8} \mathrm{H}_{4} \mathrm{O}_{4}$ for buffer solution fully shock and stewing 1 hour. Finally, the determination of absorbance values were used the solution from the chloroform layer and joined the $\mathrm{Na}_{2} \mathrm{SO}_{4}$ for dehydration. Alkaloids of Actinidia arguta were determinate by spectrophotography.

Single factor experiment

The ethanol concentration. Using ultrasound, $5 \mathrm{~g}$ of dried powder of samples were extracted with $100 \mathrm{~mL}$ ethanol with different concentrations of $40 \%, 60 \%, 70 \%, 80 \%$ and $100 \%$ at $40 \mathrm{C}$ for $30 \mathrm{~min}$.

The solid-liquid ratio. Using ultrasound, the different solid-liquid ratios of 1:15, 1:20, 1:25, 1:30 and 1:35 were extracted with concentration of $80 \%$ at $40 \mathrm{C}$ for $30 \mathrm{~min}$.

The extracting time. Using ultrasound, $5 g$ of dried powder of samples were extracted with $100 \mathrm{~mL}$ ethanol with concentration of $80 \%$ at $40 \mathrm{C}$ for different times of $20 \mathrm{~min}, 30 \mathrm{~min}, 40 \mathrm{~min}$, $50 \mathrm{~min}$ and $60 \mathrm{~min}$.

The extracting temperature. Using ultrasound, $5 \mathrm{~g}$ of dried powder of samples were extracted with $100 \mathrm{~mL}$ ethanol with concentration of $80 \%$ at different extracting temperatures of $30 \mathrm{C}, 40 \mathrm{C}$, 50C, 60C and 70C for 30 min.

Orthogonal test design. According to the results of single factor experiment, the ethanol concentration(A), the solid-liquid ratio(B), the extracting time(C) and the extracting temperature(D) are the reference factors for design $\mathrm{L}_{9}\left(3^{4}\right)$ orthogonal experimental(Table 1 ).

Table. 1 The factors and levels of orthogonal test for extraction of alkaloid from Actinidia arguta

\begin{tabular}{ccccc}
\hline Level & $\begin{array}{c}\text { Concentration(A) } \\
\%\end{array}$ & $\begin{array}{c}\text { Solid-liquid(B) } \\
\text { g:mL }\end{array}$ & $\begin{array}{c}\text { Time(C) } \\
\text { min }\end{array}$ & $\begin{array}{c}\text { Temperature(D) } \\
\text { C }\end{array}$ \\
\hline 1 & 60 & $1: 20$ & 20 & 50 \\
2 & 70 & $1: 25$ & 30 & 60 \\
3 & 80 & $1: 30$ & 40 & 70 \\
\hline
\end{tabular}

\section{Test results}

The influence of concentration of ethanol to alkaloid extraction quantity. From Fig. 1A, the best extraction yield is the $70 \%$ concentration of ethanol as the solvent and the alkaloid content achieve to $0.96 \mathrm{mg} / \mathrm{g}$. Three concentrations of ethanol $(60 \%, 70 \%$ and $80 \%)$ were chose for the orthogonal experiment.

The influence of solid-liquid ratio to alkaloid extraction quantity. From Fig. 1B, the best extraction yield is the solid-liquid ratio of $1: 25$ and the alkaloid content achieve to $1.13 \mathrm{mg} / \mathrm{g}$. Three solid-liquid ratios (1:20, 1:25 and 1:30) were chose for the orthogonal experiment.

The influence of time to alkaloid extraction quantity. From Fig. 1C, the best extraction yield is the time of $30 \mathrm{~min}$ and the alkaloid content achieve to $1.05 \mathrm{mg} / \mathrm{g}$. Three times $(20 \mathrm{~min}, 30 \mathrm{~min}$ and 40min) were chose for the orthogonal experiment.

The influence of temperature to alkaloid extraction quantity. From Fig. 1D, the best extraction yield is the temperature of $60 \mathrm{C}$ and the alkaloid content achieve to $1.13 \mathrm{mg} / \mathrm{g}$. Three temperatures (50C, 60Cand70C) were chose for the orthogonal experiment. 

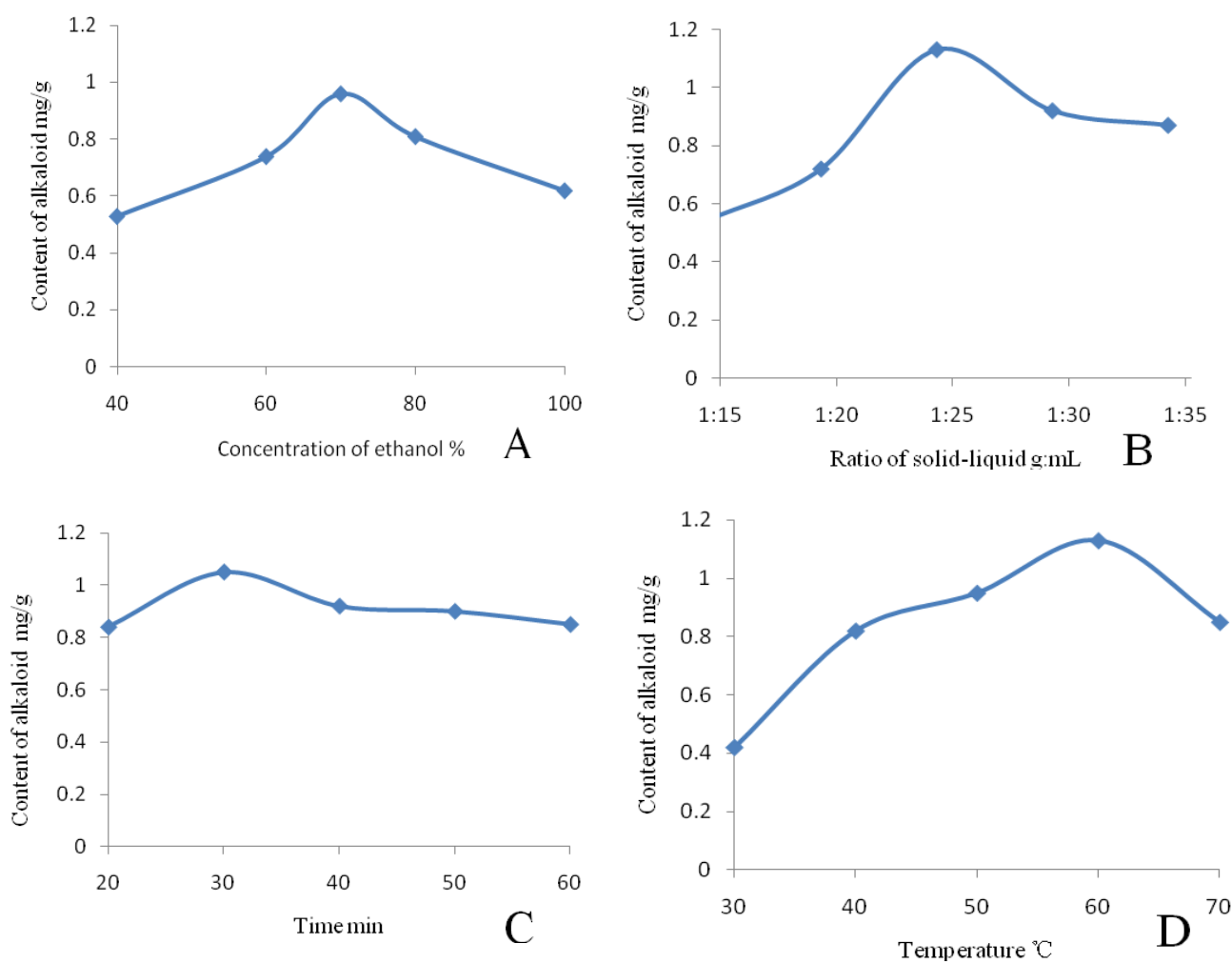

Fig.1 Alkaloid content under different ethanol concentration, solid-liquid ratio, time and temperature

The result of orthogonal experiment. From Table.2, the factors on the influence of extraction yield of alkaloid are in the order to $\mathrm{A}>\mathrm{C}>\mathrm{B}>\mathrm{D}$, namely ethanol $>$ time $>$ temperature $>$ solid-liquid ratio. The best extraction process of total alkaloid from Actinidia arguta is $A_{2} B_{3} C_{1} D_{2}$, namely $70 \%$ ethanol, solid-liquid ratio of $1: 20,20 \mathrm{~min}$ and $60 \mathrm{C}$. In this condition, the three sets of parallel tests were carried out and the alkaloid content is $1.021 \mathrm{mg} / \mathrm{g}$.

Table. 2 The result of orthogonal experiment for extraction of alkaloid from Actinidia arguta

\begin{tabular}{cccccc}
\hline No. & A & B & C & D & $\begin{array}{c}\text { Alkaloid } \\
\text { content(mg/g) }\end{array}$ \\
\hline 1 & 1 & 1 & 1 & 1 & 0.462 \\
2 & 1 & 2 & 2 & 2 & 0.654 \\
3 & 1 & 3 & 3 & 3 & 0.837 \\
4 & 2 & 1 & 2 & 3 & 0.925 \\
5 & 2 & 2 & 3 & 1 & 1.105 \\
6 & 2 & 3 & 1 & 2 & 1.076 \\
7 & 3 & 1 & 3 & 2 & 0.856 \\
8 & 3 & 2 & 1 & 3 & 0.825 \\
9 & 3 & 3 & 2 & 1 & 0.971 \\
$\mathrm{~K}_{1}$ & 1.953 & 2.705 & 2.363 & 2.438 & \\
$\mathrm{~K}_{2}$ & 3.006 & 2.484 & 2.550 & 2.586 & \\
$\mathrm{~K}_{3}$ & 2.757 & 2.884 & 2.698 & 2.587 & \\
$\mathrm{R}$ & 1.053 & 0.179 & 0.335 & 0.149 & \\
\hline
\end{tabular}

\section{Conclusion}

This study has showen that Actinidia arguta contains the more alkaloids. Ethanol concentration, solid-liquid ratio, time and temperature all have significant influence on alkaloid extraction, one of the biggest impact is the ethanol concentration. The best extraction process of total alkaloid from Actinidia arguta is $70 \%$ ethanol, solid-liquid ratio of 1:20, 20min and 60C. Experiments show that this method is feasible and good repeatability $[9,10]$. 


\section{Acknowledgement}

In this paper, the research was sponsored by The ministry of agriculture of national public welfare industry project (Project No. 200903013).

\section{References}

[1] Zhijian Lin, Liwei Yuan, Xu Guo. Chemical Composition and its Comprehensive Utilization of Actinidia arguta [J].1995 (33).

[2] Yegao Chen. Plant Chemical Composition [C].Beijing 2004. 232

[3] Deqiu Zhu, Zhongshen Liu. The Research of Actinidia arguta Anti-aging Effect [J]. Journal of Hunan Medical College, 1996 2(2) 54-57.

[4] Lijun Wu. Natural Pharmaceutical Chemistry [M].Beijing 2003 173-184

[5] Min BS, Gao J J,Nakamura N, et al. Cytotoxic Alkaloids and a Flavan from the Bulbs of Crinum Asiaticum Var. Japonicum [J]. Chem Pharm Bull, 2001 49(9) 1217-1219

[6] Chao Yu, Ming Zhang. Ultraviolet Spectrophotometric Method Determination of Total Alkaloid in Different Regions Pinellia [J]. Lishizhen Medicine and Materia Medica Research, 2002 13(2) 73

[7] Jesus G D, Juan G R, Werner H. Nordiberpene and Diterpene Alkaloids from Acoritum Voriegatum[J].Phytochemistry, 2005, 66(7) 837-846

[8] Yunchao Li, Jianping Chen, Ren Bu. The Study of Alkaloid Extraction Method and Process in Mongolian and traditional Chinese Medicine [J]. The Northern Pharmaceutical, 2013 10(12) 50-52

[9] Caixian An, Song Hai, Junmei Fu. Extraction Process Optimization of Alkaloids of Macleaya microcarpa Using Orthogonal Experiment [J]. Chinese Herbal Medicine, 2002 33(9) 804-805

[10] Tang, L., Ye, L., Lv, C., Zheng, Z., Gong, Y. and Liu, Z. Involvement of CYP3A4/5 and CYP2D6 in the metabolism of aconitine using human liver microsomes and recombinant CYP450 enzymes [J]. 2011 202, 47-54. 